\title{
Methods Dentists Use to Diagnose Primary Caries Lesions Prior to Restorative Treatment: Findings from The Dental PBRN
}

\author{
D. Brad Rindal ${ }^{5,6}$, Valeria V. Gordan ${ }^{4}$, Mark S. Litaker ${ }^{2}$, James D. Bader ${ }^{3}$, Jeffrey L. \\ Fellows ${ }^{7}$, Vibeke Qvist ${ }^{1}$, Martha C. Wallace-Dawson ${ }^{8}$, Mary L. Anderson ${ }^{5}$, and Gregg H. \\ Gilbert $^{2}$ for The DPBRN Collaborative Group \\ ${ }^{1}$ Department of Cariology and Endodontics, Royal Dental College, Copenhagen, Denmark \\ ${ }^{2}$ Department of General Dental Sciences, School of Dentistry, University of Alabama, \\ Birmingham, AL, USA \\ ${ }^{3}$ Department of Operative Dentistry and the Cecil Sheps Center for Health Services Research, \\ University of North Carolina, Chapel Hill, NC, USA \\ ${ }^{4}$ Department of Operative Dentistry, School of Dentistry, University of Florida, Gainesville, FL, \\ USA \\ ${ }^{5}$ HealthPartners Dental Group, Minneapolis, MN, USA \\ ${ }^{6}$ HealthPartners Research Foundation, Minneapolis, MN, USA \\ ${ }^{7}$ Kaiser Permanente Center for Health Research, Portland, OR, USA \\ ${ }^{8}$ Private Practitioner in Birmingham, AL, USA
}

\section{Abstract}

Objective-To (1) quantify the diagnostic techniques used by Dental Practice-Based Research Network (DPBRN) dentists before they decide to treat primary caries lesions surgically and (2) examine whether certain dentist, practice, and patient characteristics are associated with their use.

\begin{abstract}
Methods-A total of 228 DPBRN dentists recorded information on 5,676 consecutive restorations inserted due to primary caries lesions on 3,751 patients. Practitioner-investigators placed a mean of 24.9 ( $\mathrm{SD}=12.4)$ restorations. Lesions were categorized as posterior proximal, anterior proximal, posterior occlusal, posterior smooth, or anterior smooth. Techniques used to diagnose the lesion were categorized as clinical assessment, radiographs, and/or optical. Statistical analysis utilized generalized mixed-model ANOVA to account for the hierarchical structure of the data.
\end{abstract}

Results-By lesion category, the diagnostic technique combinations used most frequently were clinical assessment plus radiographs for posterior proximal (47\%), clinical assessment for anterior

\footnotetext{
(C) 2010 Elsevier Ltd. All rights reserved.

Corresponding author: D. Brad Rindal, DDS, HealthPartners Research Foundation, 8170 33rd Ave. S., M.S. 21111R, Minneapolis, MN 55425, Phone: 952-967-5026, Fax: 952-967-5022, Donald.B.Rindal@ HealthPartners.com.

For The DPBRN Collaborative Group, which includes practitioner-investigators, faculty investigators, and staff investigators who contributed to this activity (www.dpbrn.org/users/publications/default.aspx).

Declaration of interests

None of the authors declare any conflicts of interest.

Publisher's Disclaimer: This is a PDF file of an unedited manuscript that has been accepted for publication. As a service to our customers we are providing this early version of the manuscript. The manuscript will undergo copyediting, typesetting, and review of the resulting proof before it is published in its final citable form. Please note that during the production process errors may be discovered which could affect the content, and all legal disclaimers that apply to the journal pertain.
} 
proximal (51\%), clinical assessment for posterior occlusal (46\%), clinical assessment for posterior smooth (77\%), and clinical assessment for anterior smooth (80\%). Diagnostic technique was significantly associated with lesion category after adjusting for clustering in dentists $(\mathrm{p}<0.0001)$.

Conclusion-These results - obtained during actual clinical procedures rather than from questionnaire-based hypothetical scenarios — quantified the diagnostic techniques most commonly used during the actual delivery of routine restorative care. Diagnostic technique varied by lesion category and with certain practice and patient characteristics.

\section{Keywords}

Dental caries; dentists' practice patterns; diagnostic techniques and procedures

\section{Introduction}

Detection of caries lesions is crucial to their prevention and treatment ${ }^{1}$. The ideal method of detection accurately measures the depth of the caries process ${ }^{2}$, which is important in monitoring progression of active lesions and in making clinical decisions. Our current understanding ${ }^{3}$ of the caries process provides clinicians with treatment options to arrest or remineralize early lesions. If the lesion has progressed to cavitation, it is not amenable to remineralization and requires a restoration ${ }^{4}$. However, the widespread use and availability of fluoride has dramatically slowed the progression of carious lesions ${ }^{5}$ such that dentists typically detect caries at an earlier stage. In view of these changes, accurate caries detection has a critical impact on treatment decisions; incorrect diagnosis may result in incorrect treatment decisions, particularly with respect to operative intervention.

Variation among dentists in the identification and depth estimation of caries lesions is wellknown, ${ }^{6-9}$ mostly from studies of "cases" prepared by investigators. There have been few assessments of the detection techniques being used by dentists in clinical practice ${ }^{10-14}$. To learn more, we need to examine how clinicians identify lesions in their practices.

This study is a component of a broader research program being undertaken by "The Dental Practice-Based Research Network" (DPBRN, www.DPBRN.org) to investigate how dentists diagnose and treat dental caries ${ }^{15-19}$. DPBRN is a consortium of dental practices with a broad representation of practice types and treatment philosophies that conducts research across geographically dispersed regions. The objectives of this study are to (1) to quantify the diagnostic techniques used by DPBRN practitioner-investigators before they decide to treat primary caries lesions surgically, and (2) to examine whether certain dentist, practice, and patient characteristics are associated with the use of these techniques.

\section{Materials and Methods}

\section{Selection and recruitment process}

Practitioner-investigators from DPBRN who perform restorative dentistry in their practices were enrolled in this study. DPBRN comprises five regions: Alabama/Mississippi (AL/MS), Florida/Georgia (FL/GA), Minnesota dentists employed by HealthPartners Dental Group or practicing in the community (MN), Permanente Dental Associates in cooperation with Kaiser Permanente's Center for Health Research (PDA), and Denmark, Norway, and Sweden (SK) ${ }^{15}$. Practitioner-investigators in DPBRN were recruited into the network through continuing education courses and mass mailings to licensed dentists from the participating regions. As part of enrollment in DPBRN, all practitioner-investigators complete a DPBRN Enrollment Questionnaire about themselves and their practice characteristics. As part of eligibility for this particular study, all dentists completed (1) the 
Enrollment Questionnaire, (2) an Assessment of Caries Diagnosis and Caries Treatment Questionnaire, (3) training in human subjects protection, and (4) a training session with a DPBRN staff ${ }^{20}$ regional coordinator assigned to their practice. This training session discussed in detail the study protocol, data collection forms, and related details. Additional requirements varied by DPBRN region and are described elsewhere ${ }^{21}$. These questionnaires are publicly available on the DPBRN Supplement page 22 .

\section{Study design}

This cross-sectional study used a consecutive patient/restoration recruitment design. Once the study was started in a practice, every patient scheduled to have a restoration on a previously unrestored permanent tooth surface was asked to participate until 50 patients had been enrolled or a certain date had passed. If patients had multiple appointments during the study period, data were collected only at the first appointment. To broaden enrollment, we limited the number of eligible restorations to four during the patient's first appointment in the study period. A consecutive patient/restoration log form was used to record information on eligible restorations regardless of whether the patient participated in the study. All of the data collection forms used for this study is available on the DPBRN Supplement page (www.dentalpbrn.org/users/publications/supplement.aspx). The survey was pilot-tested to assess the feasibility and comprehension of each questionnaire item ${ }^{23}$.

We collected data for: (a) patient race, Hispanic/Latino ethnicity, sex, and age; (b) tooth number, surface, and primary reason for placement of the restoration (i.e., primary caries or non-carious defect); and (c) techniques used to diagnose the primary caries (i.e., probing, radiographs, transillumination, or optical technique such as Diagnodent). This study also collected data on preoperative depth, postoperative depth, and restorative materials placed. The latter results are not presented here; we limited our analyses to carious lesions involving only one surface. We considered multisurface categories, but the number of lesions in each category was small, limiting our ability to draw meaningful conclusions.

\section{Dentist-level and practice-level variables}

Dentist-level variables were available from the DPBRN Enrollment Questionnaire. In addition to DPBRN region, DPBRN dentists can also be characterized by type of practice (i.e., solo or small group private practice [SGP], large group practice [LGP], or public health practice (PHP). SGPs were defined as having no more than three dentists. LGPs were defined as having four or more dentists. PHPs were defined as receiving most of their funding from public sources. In the AL/MS region, $98 \%$ of practitioner-investigators were in SGPs, and 2\% were in PHPs. In the FL/GA region, 97\% were in SGPs, and 3\% were in PHPs. In the MN region, $90 \%$ were in LGPs, and $10 \%$ were in SGPs. In the PDA region, all were in LGPs. In the SK region, 64\% were in SGPs, and 36\% were in PHPs. The dentist's year of graduation from dental school, gender, and ethnicity were also available. Dentists were given several choices to describe their workload during the past year.

\section{Patient-level variables}

For each enrolled patient, data were collected about the patient's gender, age, race, Hispanic or Latino ethnicity, and any dental insurance or third-party coverage.

\section{Statistical analysis}

Our primary statistical analytic approach used generalized linear models (GLM) implemented with generalized estimating equations (GEE) in SAS ${ }^{\circledR}$ PROC GENMOD software to conduct analysis of variance (ANOVA) and logistic regression analysis, accounting for correlations among observations due to the hierarchical structure of the data/ 
clustering. A generalized estimating equations approach to logistic regression was used to model the associations between use of diagnostic technique and dentist-, practice-, and patient-level characteristics while simultaneously accounting for within-dentist, withinpractice, and within-patient clustering. This clustering is due to the fact that dentists/ practices enrolled numerous patients from the same practice (within-dentist clustering), and patients could have had as many as four restorations during the study (within-patient clustering). Diagnostic method use showed a median intra-class correlation coefficient (ICC) of 0.22 for clustering by dentist and practice, and 0.54 for clustering by patient within dentist and practice; accounting for the effect of clustering was essential to the validity of the statistical models. Maximum-likelihood estimates of ICCs were obtained from the GEE working correlation matrices. Ordinarily, bivariate cross-tabulations done as in Tables 2 and 3 would be tested for statistical significance using $\chi^{2}$ tests and Mantel-Haenszel $\chi^{2}$ trend tests. However, this was not appropriate in this context because of the within-class clustering. Therefore, statistical tests in Table 4 were done using GEE-based logistic regressions to account for the effect of this clustering.

Lesions were classified into five categories on the basis of the surfaces identified as involved in the restoration (i.e., posterior proximal, anterior proximal, posterior occlusal, posterior smooth surface, and anterior smooth surface). Frequencies of use of each of the techniques were tabulated by surface classification and region for all restorations. Because more than one technique could be specified, and more than a single surface could be included in a single restoration, these counts are not mutually exclusive. GEE-based ANOVA was used to compare rates of use of the diagnostic techniques among regions and surface classifications. GEE logistic regression was conducted to identify predictors of use of each of the diagnostic techniques. These analyses were restricted to restorations classified into a single surface category. Modeling was conducted separately for each of the diagnostic techniques.

Model selection was conducted within two blocks of potential predictors, representing 1) practitioner- and practice-level variables and 2) patient-level variables. Practitioner-level variables included region, gender, years since graduation from dental school $(<5,5-15,15-$ $20,>20$ ), type of practice (SGP, LGP, or PHP), and whether caries risk assessment is routinely conducted (No or no response; yes, no form used or not known if form was used; yes, using form). Patient-level variables considered were age, gender, race (white, black, American Indian/Alaska native, Asian, native Hawaiian/other Pacific islander, other), ethnicity (Hispanic/Latino, not), and whether the patient had dental insurance. Within each block, separate analyses were conducted for each potential predictor variable. Variables showing significant association at $p<0.10$ with use of a technique were then included in a multiple logistic regression model. Variables that were significant at $p<0.10$ in either of the block-level multivariable models were included in a final predictive model for the respective diagnostic technique to avoid excluding variables that might become more significant in the multivariable model.

\section{Results}

Ninety-five percent of eligible consecutive patients enrolled in the study. Table 1 shows the percentage of use of the different methods of diagnosis, alone or in combination with the other techniques, by lesion location. Diagnostic technique was significantly associated with lesion location after adjusting for clustering in dentists $(p<0.0001)$. Radiographs plus clinical assessment (47\%) and radiographs alone (40\%) were used most commonly to detect posterior proximal caries. Clinical assessment $(51 \%)$ and clinical assessment plus radiographs (29\%) were the most common detection method for anterior proximal caries. Clinical assessment only (46\%) and clinical assessment plus radiographs (41\%) were the 
most common approaches for occlusal surfaces. Clinical assessment only was used by the large majority of dentists to detect caries on posterior (77\%) and anterior smooth surfaces $(80 \%)$.

Dentist and practice characteristics potentially associated with the use of each diagnostic technique were first analyzed in a univariate model (Table 2). Variables associated at $p<0.10$ were included in the final model. Thus, practice type, use of caries risk assessment, and region were included in the final model for clinical assessment; practice type and region in the model for radiographs; and use of risk assessment and region in the model for transillumination or optical technique.

Patient characteristics that were evaluated for association with the use of a diagnostic technique are presented in Table 3. For clinical assessment, patient age and ethnicity were included in the final model; for radiographs, patient age and insurance coverage were included; and for transillumination or optical technique, patient gender was included.

Patient, dentist, and practice characteristics included in the multiple logistic regression model are presented in Table 4. Regional differences were detected in the use of clinical assessment $(p=0.0021)$ and radiographs $(p=0.0007)$. The AL/MS and FL/GA regions rely more on clinical assessment and less on radiographs than other regions. We also saw an association of region $(p=0.0189)$ and use of transillumination or optical technique. The overall use of optical technique was low (used to detect 371 lesions), and the results are difficult to summarize because of differences in cluster size.

Patient variables associated with use of diagnostic technique include age $(p<0.0001$, for radiograph), ethnicity ( $p=0.0023$, for clinical assessment), and dental insurance ( $p=0.0449$, for radiograph). Older patients are less likely to receive radiographs. Clinical assessment was listed for $91.5 \%$ of restorations in Hispanic patients vs. $81.9 \%$ of restorations in nonHispanic patients. Patients with dental insurance are less likely to receive radiographs.

\section{Discussion}

These results further illuminate the diagnostic techniques used by dentists in daily practice to detect initial caries on a previously unrestored surface. They also provide insight into patient and provider characteristics that may influence the use of these techniques. Regional differences in the application of the clinical assessment would suggest differences in training and accepted standards of care. We need to be careful in drawing conclusions about the use of transillumination or optical techniques, because they are used infrequently and usually in combination with other techniques.

Use of radiographs is related to DPBRN region, age of the patient, and dental insurance benefits. It is possible that older patients have a longer dental history for the dentist to consider when deciding if a radiograph is needed to detect caries in areas not observed visually. Dental insurance determines the cost to the patient for radiographs; the counterintuitive observation that patients with dental insurance are less likely to receive radiographs suggests that benefit limitations common to dental insurance policies may influence provider and patient decisions regarding radiographs. The regional differences might be related to teaching and peer norms regarding the prescribing of radiographs.

Clinical assessments and radiographs continue to be the primary caries detection methods employed by dentists in daily practice. Despite the marketing of diagnostic tools such as DIAGNOdent, they are used at very low rates by dentists enrolled in The DPBRN. As new diagnostic techniques become available in the future, practice-based research networks will afford us the opportunity to examine their adoption in daily practice. A recent systematic 
review of current evidence presented in the literature concluded that utilization of a combination of visual-tactile and radiographic evidence is still the best caries diagnostic technique. Current practice is consistent with current evidence ${ }^{24}$.

\section{Conclusion}

These results - obtained during actual clinical procedures rather than from questionnairebased hypothetical scenarios - quantified the diagnostic techniques most commonly used by practicing dentist in real-world setting during the actual delivery of routine restorative care. We identified significant regional differences in the utilization of the various diagnostic techniques. These regional differences may be due to differences in dental education and community practice norms. Patient age, gender and having dental insurance are also associated with the use of diagnostic technique.

\section{References}

1. Bader JD, Shugars DA. A systematic review of the performance of a laser fluorescence device for detecting caries. Journal of American Dental Association. 2004; 135(10):1413-26.

2. Pitts NB. Modern concepts of caries measurement. Journal of Dental Research. 2004; 83(Spec No C):C43-7. [PubMed: 15286121]

3. Bader JD, Shugars DA. The evidence supporting alternative management strategies for early occlusal caries and suspected occlusal dentinal caries. Journal of Evidence Based Dental Research. 2006; 6(1):91-100.

4. Pitts NB, Stamm JW. International Consensus Workshop on Caries Clinical Trials (ICW-CCT)-final consensus statements: agreeing where the evidence leads. Journal of Dental Research. 2004; 83(Spec No C):C125-8. [PubMed: 15286139]

5. Zandona AF, Zero DT. Diagnostic tools for early caries detection. Journal of American Dental Association. 2006; 137(12):1675-84.

6. Lewis DW, Pharoah MJ, El-Mowafy O, Ross DG. Restorative certainty and varying perceptions of dental caries depth among dentists. Journal of Public Health Dentistry. 1997; 57(4):243-5. [PubMed: 9558628]

7. el-Mowafy OM, Lewis DW. Restorative decision making by Ontario dentists. Journal of the Canadian Dental Association. 1994; 60(4):305-10. 13-6. [PubMed: 8037797]

8. Winn DM, Brunelle JA, Selwitz RH, Kaste LM, Oldakowski RJ, Kingman A, et al. Coronal and root caries in the dentition of adults in the United States, 1988-1991. Journal of Dental Research. 1996; 75(Spec No):642-51. [PubMed: 8594088]

9. Mettes TG, van der Sanden WJ, Mokkink HG, Wensing M, Grol RP, Plasschaert AJ. Routine oral examinations in primary care: which predictors determine what is done? A prospective clinical case recording study. Journal of Dentistry. 2008; 36(6):435-43. [PubMed: 18406034]

10. Gilbert GH, Bader JD, Litaker MS, Shelton BJ, Duncan RP. Patient-level and practice-level characteristics associated with receipt of preventive dental services: 48-month incidence. Journal of Public Health Dentistry. 2008; 68(4):209-17. [PubMed: 18248347]

11. Gilbert GH, Weems RA, Litaker MS, Shelton BJ. Practice characteristics associated with patientspecific receipt of dental diagnostic radiographs. Health Services Research. 2006; 41(5):1915-37. [PubMed: 16987308]

12. Domejean-Orliaguet S, Leger S, Auclair C, Gerbaud L, Tubert-Jeannin S. Caries management decision: influence of dentist and patient factors in the provision of dental services. Journal of Dentistry. 2009; 37(11):827-34. [PubMed: 19628326]

13. Zadik Y, Levin L. Clinical decision making in restorative dentistry, endodontics, and antibiotic prescription. Journal of Dental Education. 2008; 72(1):81-6. [PubMed: 18172239]

14. Mettes TG, van der Sanden WJ, Mokkink HG, Wensing M, Grol RP, Plasschaert AJ. Routine oral examination: clinical performance and management by general dental practitioners in primary care. European Journal of Oral Sciences. 2007; 115(5):384-9. [PubMed: 17850427] 
15. Gilbert GH, Williams OD, Rindal DB, Pihlstrom DJ, Benjamin PL, Wallace MC. The creation and development of The Dental pPractice-Based Research Network. Journal of American Dental Association. 2008; 139(1):74-81.

16. Makhija SK, Gilbert GH, Rindal DB, Benjamin P, Richman JS, Pihlstrom DJ, et al. Practices participating in a dental PBRN have substantial and advantageous diversity even though as a group they have much in common with dentists at large. BioMed Central Oral Health. 2009; 9:26. [PubMed: 19832991]

17. Gordon V, Bader JD, Garvan C, Richman JS, Qvist V, Fellows J, et al. Restorative treatment thresholds for occlusal primary caries by dentists in The Dental PBRN. Journal of the American Dental Association. (in press).

18. Gordon V, Garvan C, Heft M, Fellows J, Qvist V, Rindal DB, et al. Restorative treatment thresholds for interproximal primary caries based on radiographic images: findings from The Dental PBRN. General Dentistry. 2009; 57(6):654-63. [PubMed: 19906618]

19. Nascimento M, Gordon V, Mjor I, Qvist V, Litaker MS, Williams OD, et al. Reasons for placement of restorations on previously unrestored tooth surfaces by Dental PBRN dentists. Journal of the American Dental Association. (in press).

20. Gilbert GH, Qvist V, Moore SD, Rindal DB, Fellows JL, Gordan VV, et al. Institutional review board and regulatory solutions in The Dental PBRN. Journal of Public Health Dentistry. 2009

21. Supplements to specific DPBRN publications. Dental Practice-Based Research Network; at http://www.dentalpbrn.org/users/publications/Supplement.aspx

22. Gordan VV, Garvan CW, Heft MW, Fellows JL, Qvist V, Rindal DB, et al. Restorative treatment thresholds for interproximal primary caries based on radiographic images: findings from the Dental Practice-Based Research Network. General Dentistry. 2009; 57(6):654-63. quiz 64-6, 595, 680. [PubMed: 19906618]

23. Ewoldsen N, Koka S. There are no clearly superior methods for diagnosing, predicting, and noninvasively treating dental caries. Journal of Evidence Based Dental Research. 2010; 10(1):167. 


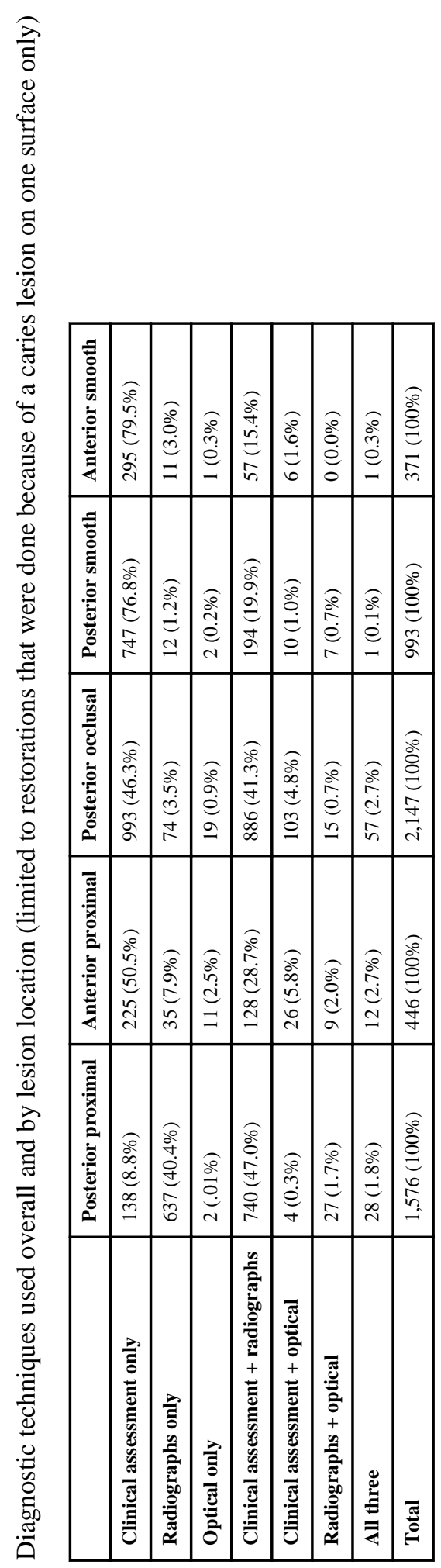

J Dent. Author manuscript; available in PMC 2012 January 27. 
Table 2

Association ( $p$ value) of dentist and practice characteristics with use of diagnostic technique in one variable models

\begin{tabular}{|l|c|c|c|}
\hline \multirow{2}{*}{ Characteristic } & \multicolumn{3}{|c|}{ p value } \\
\cline { 2 - 4 } & Clinical assessment & Radiograph & Transillumination or optical technique \\
\hline Years since graduation & 0.2307 & 0.4687 & 0.1304 \\
\hline Gender of dentist & 0.1135 & 0.2984 & 0.9965 \\
\hline Race/ethnicity of dentist & 0.5893 & 0.5127 & NOTE \\
\hline Practice type (solo, group, public) & 0.0187 & 0.0007 & 0.1358 \\
\hline Caries risk assessment & 0.0531 & 0.1125 & 0.0159 \\
\hline Region & 0.0017 & 0.0010 & 0.0337 \\
\hline
\end{tabular}

NOTE: Estimation algorithm failed. Dentist race distribution is sparse ( $88.8 \%$ white) and relatively small number (341) of uses of optical technique (307 of which were done by white dentists). 
Table 3

Association ( $p$ value) of patient characteristics with use of diagnostic technique in one variable models

\begin{tabular}{|l|c|c|c|}
\hline \multirow{2}{*}{ characteristic } & \multicolumn{3}{|c|}{$p$ value } \\
\cline { 2 - 4 } & Clinical assessment & Radiograph & Transillumination or optical technique \\
\hline Age & 0.0529 & $<0.0001$ & 0.5783 \\
\hline Gender & 0.9231 & 0.7822 & 0.0633 \\
\hline Race & 0.8150 & 0.3633 & 0.6636 \\
\hline Ethnicity & 0.0022 & 0.2707 & 0.9742 \\
\hline Insurance & 0.8411 & 0.1094 & 0.3852 \\
\hline
\end{tabular}


Table 4

Association of dentist, practice, and patient characteristics with use of diagnostic technique in final models (only statistically significant $p$-values are provided)

\begin{tabular}{|l|l|l|l|}
\hline Characteristic & Clinical assessment & Radiograph & Transillumination or optical technique \\
\hline Years since graduation from dental school & & & \\
\hline Gender of dentist & & & \\
\hline Practice type (SGP, LGP, PHP) & & & \\
\hline Dentist uses caries risk assessment & & & \\
\hline Region & 0.0021 & 0.0007 & \\
\hline Patient age & & $<0.0001$ & \\
\hline Patient gender & & & \\
\hline Patient race & & & \\
\hline Patient ethnicity & 0.0023 & & \\
\hline Whether patient has dental insurance & & 0.0671 \\
\hline
\end{tabular}

LGP: large group practice; PHP: public health practice; SGP: small group practice. 